hep-ph/0102320

DFTT 3/2001

February 27, 2001

\title{
Lepton Numbers in the framework of Neutrino Mixing
}

\author{
S.M. Bilenky \\ Joint Institute for Nuclear Research, Dubna, Russia, and \\ INFN, Sez. di Torino, and Dip. di Fisica Teorica, Univ. di Torino, I-10125 Torino, Italy \\ and \\ C. Giunti \\ INFN, Sez. di Torino, and Dip. di Fisica Teorica, Univ. di Torino, I-10125 Torino, Italy
}

\begin{abstract}
In this short review we discuss the notion of lepton numbers. The strong evidence in favor of neutrino oscillations obtained recently in the Super-Kamiokande atmospheric neutrino experiment and in solar neutrino experiments imply that the law of conservation of family lepton numbers $L_{e}, L_{\mu}$ and $L_{\tau}$ is strongly violated. We consider the states of flavor neutrinos $\nu_{e}, \nu_{\mu}$ and $\nu_{\tau}$ and we discuss the evolution of these states in space and time in the case of non-conservation of family lepton numbers due to the mixing of light neutrinos. We discuss and compare different flavor neutrino discovery experiments. We stress that experiments on the search for $\nu_{\mu} \rightarrow \nu_{\tau}$ and $\nu_{e} \rightarrow \nu_{\tau}$ oscillations demonstrated that the flavor neutrino $\nu_{\tau}$ is a new type of neutrino, different from $\nu_{e}$ and $\nu_{\mu}$. In the case of neutrino mixing, the lepton number (only one) is connected with the nature of massive neutrinos. Such conserved lepton number exist if massive neutrinos are Dirac particles. We review possibilities to check in future experiments whether the conserved lepton number exists.
\end{abstract}




\section{Introduction}

The strong evidence in favor of neutrino masses and mixing that was obtained recently in the Super-Kamiokande atmospheric neutrino experiment [四] opened a new epoch in neutrino physics. Evidence in favor of neutrino mixing was also obtained in all solar neutrino experiments: Homestake [2], Kamiokande [3], GALLEX [4], SAGE [5], Super-Kamiokande [6], GNO [7]. Indications in favor of $\nu_{\mu} \rightarrow \nu_{e}$ oscillations were found in the accelerator LSND experiment [8].

All these data can be explained in terms of neutrino oscillations, if the masses of neutrinos are different from zero and the fields of massive neutrinos enter in the standard $\mathrm{CC}$ and $\mathrm{NC}$ interaction Lagrangian

$$
\begin{array}{ll}
\mathcal{L}_{I}^{\mathrm{CC}}=-\frac{g}{2 \sqrt{2}} j_{\alpha}^{\mathrm{CC}} W^{\alpha}+\text { h.c. }, & j_{\alpha}^{\mathrm{CC}}=2 \sum_{\ell=e, \mu, \tau} \overline{\nu_{\ell L}} \gamma_{\alpha} \ell_{L}+\ldots \\
\mathcal{L}_{I}^{\mathrm{NC}}=-\frac{g}{2 \cos \theta_{W}} j_{\alpha}^{\mathrm{NC}} Z^{\alpha}+\text { h.c. }, & j_{\alpha}^{\mathrm{NC}}=\sum_{\ell=e, \mu, \tau} \overline{\nu_{\ell L}} \gamma_{\alpha} \nu_{\ell L}+\ldots
\end{array}
$$

in the mixed form円

$$
\nu_{\ell L}=\sum_{i=1}^{3} U_{\ell i} \nu_{i L} .
$$

Here $\nu_{i}$ is the field of the neutrino with mass $m_{i}$ and $U$ is the mixing matrix.

In the framework of theories with massless neutrinos it was customary to introduce the family lepton numbers $L_{e}, L_{\mu}$ and $L_{\tau}$, correspondingly, for the pairs $\left(\nu_{e}, e^{-}\right),\left(\nu_{\mu}, \mu^{-}\right)$and $\left(\nu_{\tau}, \tau^{-}\right)$. The observation of neutrino oscillations clearly demonstrates that family lepton numbers are not conserved.

In Section 2 we consider the states of flavor neutrinos and the evolution of these states in vacuum. In Section 3 we discuss and compare different flavor neutrino discovery experiments. In Section 4 we review the concept of lepton number in the framework of the theory of neutrino masses and mixing.

\section{Flavor neutrino states}

In this Section we consider, in the framework of neutrino mixing:

1. Decays in which family lepton numbers are not conserved (like $\mu^{+} \rightarrow e^{+}+\gamma, \mu^{+} \rightarrow$ $e^{+}+e^{-}+e^{+}$and others).

2. Flavor neutrino states.

3. Transitions between flavor neutrinos in vacuum (neutrino oscillations).

\footnotetext{
${ }^{1}$ In order to describe all existing neutrino oscillation data, including LSND data, it is necessary to assume that there are transitions of flavor neutrinos $\nu_{\ell}$ into sterile states (see, for example, [9]). We will not consider here this possibility.
} 
If there is neutrino mixing (Eq. (3)), processes of transitions between leptons of different families, as $\mu^{+} \rightarrow e^{+}+\gamma, \mu^{+} \rightarrow e^{+}+e^{-}+e^{+}$and others, become possible. Let us consider, for example, the decay $\mu^{+} \rightarrow e^{+}+\gamma$ (see the diagrams in Fig. (1). In the simplest case of mixing of two neutrinos with masses $m_{1}$ and $m_{2}$ the ratio $R$ of the probability of $\mu^{+} \rightarrow e^{+}+\gamma$ decay to the probability of the decay $\mu^{+} \rightarrow e^{+}+\nu_{e}+\bar{\nu}_{\mu}$ is given by [10, 11, 12]

$$
R=\frac{3 \alpha}{128 \pi}\left(\frac{\Delta m^{2}}{m_{W}^{2}}\right)^{2} \sin ^{2} 2 \vartheta,
$$

where $m_{W}$ is the mass of the $W$ boson, $\Delta m^{2} \equiv m_{2}^{2}-m_{1}^{2}$ and $\vartheta$ is the neutrino mixing angle.

The value of $\Delta m^{2} / m_{W}^{2}$ in Eq. (田) is

$$
\frac{\Delta m^{2}}{m_{W}^{2}} \simeq 1.5 \times 10^{-22} \frac{\Delta m^{2}}{\mathrm{eV}^{2}} .
$$

For $\Delta m^{2} \lesssim 1 \mathrm{eV}^{2}$, for the ratio $R$ we have

$$
R \lesssim 1.2 \times 10^{-48} .
$$

Thus, though the processes $\mu^{+} \rightarrow e^{+}+\gamma, \mu^{+} \rightarrow e^{+}+e^{-}+e^{+}$and others are in principle allowed in the case of neutrino mixing, it is practically impossible to observe them[?

The strong suppression of the probability of processes like $\mu^{+} \rightarrow e^{+}+\gamma$ is due to the fact that the coefficient in Eq. (50) is very small. As we will see later, in the case of neutrino oscillations the corresponding coefficient, $L / E$ ( $E$ is the neutrino energy and $L$ is the distance between neutrino source and detector), can be many orders of magnitude larger. This is the main reason why effects of violation of the law of conservation of lepton numbers can be revealed in neutrino oscillation experiments

Let us consider now, in the framework of neutrino mixing, the state $\left|\nu_{\ell}\right\rangle$ of a flavor neutrino produced in the $\mathrm{CC}$ weak decay process

$$
A \rightarrow B+\ell^{+}+\nu_{\ell} .
$$

For example, in the case of neutrinos produced in nuclear $\beta^{+}$decay, $A=\mathcal{N}(A, Z), B=$ $\mathcal{N}(A, Z-1), \ell=e$.

If there is neutrino mixing, the flavor neutrino state $\left|\nu_{\ell}\right\rangle$ is a superposition of states of massive neutrinos $\nu_{i}$ :

$$
\left|\nu_{\ell}\right\rangle=\sum_{i}\left|\nu_{i}\right\rangle\left\langle\ell^{+}, \nu_{i}, B|S| A\right\rangle,
$$

where $\left\langle\ell^{+}, \nu_{i}, B|S| A\right\rangle$ is the relevant element of the $S$ matrix.

\footnotetext{
${ }^{2}$ If the violation of the law of conservation of lepton numbers is due to other mechanisms, as supersymmetry, the probability of the decay $\mu^{+} \rightarrow e^{+}+\gamma$ and other similar processes can be much larger than in the case of neutrino mixing (see 13, 14, 15, 16]).

${ }^{3}$ In the case of neutrinoless double- $\beta$ decay of nuclei $\mathcal{N}(A, Z) \rightarrow \mathcal{N}(A, Z+2)+e^{-}+e^{-}$the suppression of the decay probability is less strong than in the decay $\mu^{+} \rightarrow e^{+}+\gamma$ and similar processes. We consider neutrinoless double- $\beta$ decay in Section 4 .
} 
From the data of laboratory experiments and astrophysical observations we know that neutrino masses are very small (see [17]),

$$
m_{i} \lesssim \text { few eV }
$$

Since in order to be detected in present-day experiments neutrinos must have energy $E \gtrsim$ $0.1 \mathrm{MeV}$, we have $m_{i}^{2} / E^{2} \ll 1$. Thus, the kinematical dependence of the matrix element $\left\langle\ell^{+}, \nu_{i}, B|S| A\right\rangle$ on neutrino masses can be neglected with very good approximation, leading to

$$
\left.\left\langle\ell^{+}, \nu_{i}, B|S| A\right\rangle \simeq U_{\ell i}^{*}\left\langle\ell^{+}, \nu_{\ell}, B|S| A\right\rangle\right|_{m_{i}=0} .
$$

Here $\left.\left\langle\ell^{+}, \nu_{\ell}, B|S| A\right\rangle\right|_{m_{i}=0}$ is the matrix element evaluated in the Standard Model, with zero neutrino masses. From Eqs. (8) and (10), the normalized state describing the flavor neutrino produced in the decay process (7) is

$$
\left|\nu_{\ell}\right\rangle=\sum_{i} U_{\ell i}^{*}\left|\nu_{i}\right\rangle
$$

Neutrinos $\nu_{i}$ with mass $m_{i}$ are produced in standard weak decays in states with lefthanded as well as right-handed helicities. However, in the Standard Model the probability to produce a neutrino $\nu_{i}$ in a state with right-handed helicity is negligibly small because it is proportional to $m_{i}^{2} / E^{2}$. Thus, $\nu_{i}$ 's and, consequently, flavor neutrinos $\nu_{\ell}$ are produced in standard weak interaction processes in almost pure left-handed states.

The state of antineutrino $\left|\bar{\nu}_{\ell}\right\rangle$, the particle that is produced in a CC weak process together with a $\ell^{-}$, is given by

$$
\left|\bar{\nu}_{\ell}\right\rangle=\sum_{i} U_{\ell i}\left|\bar{\nu}_{i}\right\rangle
$$

The state vector $\left|\bar{\nu}_{i}\right\rangle$ describes antineutrinos with right-handed helicity in the case of Dirac $\nu_{i}$ or neutrinos with right-handed helicity in the case of Majorana $\nu_{i}$. Hence, the state vector $\left|\bar{\nu}_{\ell}\right\rangle$ describes neutrinos with right-handed helicity.

In the general case of $\mathrm{CP}$ violation in the lepton sector, there are phases in the neutrino mixing matrix $U$. Therefore, the states $\left|\nu_{\ell}\right\rangle$ and $\left|\bar{\nu}_{\ell}\right\rangle$ differ not only by helicity, but also by the sign of the CP-violating phases. The violation of CP in the lepton sector can be revealed through the investigation of neutrino oscillations (see, for example, [12, 9]).

In oscillation experiments neutrinos are detected at some distance from the source. Neutrinos produced as flavor neutrinos $\nu_{\ell}$ are described at the source by the state (11). Taking into account the evolution in space and time, the neutrino beam at the distance $\vec{x}$ from the source and at the time $t$ after production is described by the state

$$
\sum_{i} e^{i\left(\vec{p}_{i} \cdot \vec{x}-E_{i} t\right)} U_{\ell i}^{*}\left|\nu_{i}\right\rangle
$$

where $\vec{p}_{i}$ is the three-momentum of the massive neutrino $\nu_{i}$ and $E_{i}=\sqrt{\left|\vec{p}_{i}\right|^{2}+m_{i}^{2}}$ is its energy. 
Let us consider an experiment in which neutrinos described by the state (13) are detected through the observation of the CC process

$$
\nu_{\ell^{\prime}}+N \rightarrow \ell^{\prime}+X
$$

Here $N$ is a target nucleon, $\ell^{\prime}$ is the final lepton and $X$ represents final hadrons. The amplitude of the process (14) is proportional to

$$
\sum_{i}\left\langle\ell^{\prime}, X|S| N, \nu_{i}\right\rangle e^{i\left(\vec{p}_{i} \cdot \vec{x}-E_{i} t\right)} U_{\ell i}^{*}
$$

As in the case of neutrino production, for ultra-relativistic neutrinos the contribution of neutrino masses to the matrix element in Eq. (15) can be neglected with very good approximation:

$$
\left.\left\langle\ell^{\prime}, X|S| N, \nu_{i}\right\rangle \simeq U_{\ell^{\prime} i}\left\langle\ell^{\prime}, X|S| N, \nu_{\ell^{\prime}}\right\rangle\right|_{m_{i}=0},
$$

where $\left.\left\langle\ell^{\prime}, X|S| N, \nu_{\ell^{\prime}}\right\rangle\right|_{m_{i}=0}$ is the matrix element evaluated in the Standard Model, with massless neutrinos. Therefore, the probability amplitude to observe a flavor neutrino $\nu_{\ell^{\prime}}$ at the distance $\vec{x}$ from the source and at the time $t$ after production of a flavor neutrino $\nu_{\ell}$ is given by

$$
\mathcal{A}_{\nu_{\ell} \rightarrow \nu_{\ell^{\prime}}}(\vec{x}, t)=\sum_{i} U_{\ell^{\prime} i} e^{i\left(\vec{p}_{i} \cdot \vec{x}-E_{i} t\right)} U_{\ell i}^{*}
$$

From this expression it is clear that transitions between different flavor neutrinos can take place only if the following two conditions are satisfied:

1. The matrix $U$ is non-diagonal.

2. The phase factors $e^{i\left(\vec{p}_{i} \cdot \vec{x}-E_{i} t\right)}$ for different massive neutrinos $\nu_{i}$ are different.

If neutrinos are massless, $\vec{p}_{i}=\vec{p}$ and $E_{i}=E$, leading to the transition probability

$$
P_{\nu_{\ell} \rightarrow \nu_{\ell^{\prime}}}(\vec{x}, t)=\left|\mathcal{A}_{\nu_{\ell} \rightarrow \nu_{\ell^{\prime}}}(\vec{x}, t)\right|^{2}=\delta_{\ell \ell^{\prime}} .
$$

In general, using the unitarity of the neutrino mixing matrix, we have

$$
0 \leq P_{\nu_{\ell} \rightarrow \nu_{\ell^{\prime}}}(\vec{x}, t) \leq \sum_{i}\left|U_{\ell^{\prime} i}\right|^{2} \sum_{k}\left|U_{\ell k}\right|^{2}=1
$$

Let us enumerate the neutrino masses in such a way that

$$
m_{1}<m_{2}<m_{3}
$$

Taking into account the unitarity of the mixing matrix $U$, the amplitude (17) of $\nu_{\ell} \rightarrow \nu_{\ell^{\prime}}$ transitions can be written in the form

$$
\mathcal{A}_{\nu_{\ell} \rightarrow \nu_{\ell^{\prime}}}(x)=e^{i\left(\vec{p}_{1} \cdot \vec{x}-E_{1} t\right)}\left\{\delta_{\ell \ell^{\prime}}+\sum_{i} U_{\ell i}^{*} U_{\ell^{\prime} i}\left[e^{\left.i\left(\vec{p}_{i}-\vec{p}_{1}\right) \cdot \vec{x}-i\left(E_{i}-E_{1}\right) t\right)}-1\right]\right\} .
$$


It is obvious that the common phase $e^{i\left(\vec{p}_{1} \cdot \vec{x}-E_{1} t\right)}$ does not enter into the expression for the transition probability. Taking into account the fact that the neutrino is detected only if its three-momentum is aligned along $\vec{x}$ (i.e. $\vec{p}=|\vec{p}| \vec{x} /|\vec{x}|)$, the phase difference $\phi_{i 1}=\left(\vec{p}_{i}-\vec{p}_{1}\right)$. $\vec{x}-\left(E_{i}-E_{1}\right) t$ in Eq. (21) can be written as

$$
\phi_{i 1}=\left(p_{i}-p_{1}\right) x-\left(E_{i}-E_{1}\right) t
$$

where $x \equiv|\vec{x}|$ and $p_{i} \equiv\left|\vec{p}_{i}\right|$. Furthermore, we have

$$
\phi_{i 1}=\frac{p_{i}^{2}-p_{1}^{2}}{p_{i}+p_{1}} x-\left(E_{i}-E_{1}\right) t=\left(\phi_{i 1}\right)_{\mathrm{st}}-\left(E_{i}-E_{1}\right)\left(t-\frac{E_{i}+E_{1}}{p_{i}+p_{1}} x\right),
$$

where

$$
\left(\phi_{i 1}\right)_{\mathrm{st}}=-\frac{\Delta m_{i 1}^{2} x}{p_{i}+p_{1}} \simeq-\frac{\Delta m_{i 1}^{2} x}{2 E}
$$

with $\Delta m_{i j}^{2} \equiv m_{i}^{2}-m_{j}^{2}$, is the standard expression for the phase difference (see, for example, [18, 12, 19, 9]). Here $E$ is the neutrino energy given by the kinematics of the production process neglecting neutrino masses. The second term on the right-hand side of Eq. (23) is much smaller than $\left(\phi_{i 1}\right)_{\text {st }}$. Indeed, the kinematics of the production process implies that

$$
E_{i}-E_{1} \sim \frac{\Delta m_{i 1}^{2}}{E}
$$

Since the velocity of the neutrino signal is equal to the velocity of light minus a correction of the order $m_{i}^{2} / E^{2}$, we have

$$
\left(t-\frac{E_{i}+E_{1}}{p_{i}+p_{1}} x\right) \sim \frac{m_{i}^{2}}{E^{2}} x
$$

Therefore, the second term on the right-hand side of Eq. (23) is of the order

$$
\left|\left(\phi_{i 1}\right)_{\mathrm{st}}\right| \frac{m_{i}^{2}}{E^{2}} \ll\left|\left(\phi_{i 1}\right)_{\mathrm{st}}\right|
$$

and can be neglectedf.

The probability of $\nu_{\ell} \rightarrow \nu_{\ell^{\prime}}$ transitions is given by

$$
P_{\nu_{\ell} \rightarrow \nu_{\ell^{\prime}}}(L)=\left|\delta_{\ell \ell^{\prime}}+\sum_{i} U_{\ell i}^{*} U_{\ell^{\prime} i}\left(e^{-i \frac{\Delta m_{i 1}^{2} L}{2 E}}-1\right)\right|^{2}
$$

where $L=x$ is the distance between neutrino source and detector.

\footnotetext{
${ }^{4}$ Let us stress that we did not assume the equality of momenta or equality of energies of massive neutrinos $\nu_{i}$. Such assumptions are often discussed in literature (see [20, 21, 22 and references therein). The wave packet treatment of neutrino transitions gives the same result [23, 24].
} 
The transition probability depends on the quantity $L / E$ that is determined by the experimental conditions. If the quantity $L / E$ is so small that for all $\Delta m_{i 1}^{2}$

$$
\frac{\Delta m_{i 1}^{2} L}{2 E} \ll 1
$$

then $P\left(\nu_{\ell} \rightarrow \nu_{\ell^{\prime}}\right) \simeq \delta_{\ell \ell^{\prime}}$ and violation of the law of conservation of family lepton numbers cannot be observed. A violation of this law can be observed only if the quantity $L / E$ is large enough so that for at least one neutrino mass-squared difference, say $\Delta m^{2}$,

$$
\frac{\Delta m^{2} L}{2 E} \gtrsim 1
$$

This condition can be rewritten as

$$
2.5 \frac{L(\mathrm{~m})}{E(\mathrm{MeV})} \Delta m^{2}\left(\mathrm{eV}^{2}\right) \gtrsim 1
$$

where $L(\mathrm{~m})$ is the source-detector distance in meters, $E(\mathrm{MeV})$ is neutrino energy in $\mathrm{MeV}$, and $\Delta m^{2}\left(\mathrm{eV}^{2}\right)$ is the neutrino mass-squared difference in $\mathrm{eV}^{2}$. In short-baseline neutrino oscillation experiments $L / E \sim 10^{-2}-10^{2}$, in long-baseline neutrino oscillation experiments $L / E \sim 10^{2}-10^{3}$, in atmospheric neutrino experiments $L / E \sim 10^{2}-10^{4}$, and in solar neutrino experiments $L / E \sim 10^{10}-10^{11}$, leading to a sensitivity to $\Delta m^{2} \sim 10^{-2}-10^{2} \mathrm{eV}^{2}$, $10^{-3}-10^{-2} \mathrm{eV}^{2}, 10^{-4}-10^{-2} \mathrm{eV}^{2}, 10^{-11}-10^{-10} \mathrm{eV}^{2}$, respectively.

In conclusion of this section, let us stress that in the case of mixing of neutrinos with small masses, flavor neutrinos and antineutrinos are not quanta of the $\nu_{e}, \nu_{\mu}$ and $\nu_{\tau}$ fields 25. In other words, $\nu_{e}, \nu_{\mu}$ and $\nu_{\tau}$ are not fields of particles.

The neutrinos $\nu_{e}, \nu_{\mu}, \nu_{\tau}$ (antineutrinos $\bar{\nu}_{e}, \bar{\nu}_{\mu}, \bar{\nu}_{\tau}$ ), are produced in CC weak decays together with, correspondingly, $e^{+}, \mu^{+}, \tau^{+}\left(e^{-}, \mu^{-}, \tau^{-}\right)$, and produce, correspondingly, $e^{-}$, $\mu^{-} \tau^{-}\left(e^{+}, \mu^{+}, \tau^{+}\right)$in $\mathrm{CC}$ processes of the interaction with nucleon etc. These neutrinos carry the flavor of corresponding leptons and their appropriate names are flavor neutrinos ?.

The states of flavor neutrinos (antineutrinos) are the superpositions of states of neutrinos with definite masses and negative helicity (positive helicity). Thus, flavor neutrinos do not have definite mass.

The investigation of neutrino oscillations is the most sensitive method to reveal the violation of the law of conservation of family lepton numbers (see [18, 12, 19, 9]).

5 There is no difference of principle between neutrino mixing and quark mixing. It is obvious that, for example, in the quark case

$$
d_{L}^{\prime}=\sum_{q=d, s, b} V_{u q} q_{L}
$$

is not a particle field but the combination of the left-handed components of the $d, s$ and $b$ fields.

${ }^{6}$ Sometimes states of flavor neutrinos are called eigenstates of weak interactions. We do not think that this name reflect the real content of the notion of a flavor neutrino state. 


\section{Flavor neutrino discovery experiments}

In this section we discuss and compare different flavor neutrino discovery experiments.

As it is well known, the electron neutrino $\nu_{e}$ was discovered by C.L. Cowan and F. Reines in the fifties [26, 27, 28]. In 1962, in the Brookhaven experiment of Lederman, Schwartz, Steinberger et al. [29] the second flavor neutrino $\nu_{\mu}$ was discovered. In 2000 the tau neutrino $\nu_{\tau}$ has been directly detected in the DONUT experiment [30].

In the Cowan and Reines experiment, electron (anti)neutrinos have been detected through the observation of the process

$$
\bar{\nu}_{e}+p \rightarrow e^{+}+n
$$

with antineutrinos from the powerful Savannah River reactor.

In a reactor $\bar{\nu}_{e}$ 's are produced in a chain of $\beta$-decays of radioactive neutron-rich nuclei, products of the fission of ${ }^{235} \mathrm{U}$ and ${ }^{238} \mathrm{U}$. The energy of reactor antineutrinos is less than about $10 \mathrm{MeV}$. About $2 \times 10^{14} \bar{\nu}_{e}$ 's are emitted per second per KW. The power of the Savannah River reactor was $\simeq 2300 \mathrm{MW}$ (th). Thus, about $2.3 \times 10^{20} \bar{\nu}_{e}$ 's per second were emitted by the reactor. The flux of $\bar{\nu}_{e}$ 's in the Cowan and Reines experiment was $\simeq 10^{13} \mathrm{~cm}^{-2} \mathrm{~s}^{-1}$.

As it is well known, the hypothesis of the existence of neutrino was put forward in 1930 by W. Pauli in order to solve the problem of continuous $\beta$-spectra and the problem of the spin and statistics of some nuclei (like ${ }^{14} N$ ). In 1933 E. Fermi assumed that an electron and an antineutrino are produced in the process

$$
n \rightarrow p+e^{-}+\bar{\nu}_{e}
$$

and proposed the first Hamiltonian of $\beta$-decay.

It is a direct consequence of quantum field theory that an $\bar{\nu}_{e}$ that is produced in $\beta$-decay together with an electron must produce a positron in the process (32). Moreover, if the interaction responsible for the decay of the neutron is known, one can connect the cross section of the process (32) at the small reactor energies with the lifetime of the neutron. Neglecting small corrections due to neutron recoil, the total cross section of the process (32) is given by (see, for example, [31])

$$
\sigma\left(\bar{\nu}_{e} p \rightarrow e^{+} n\right)=\frac{2 \pi^{2}}{m_{e}^{5} f \tau_{n}} p_{e} E_{e} .
$$

Here $E_{e}=E-\left(m_{n}-m_{p}\right)$ is the energy of the positron ( $E$ is the antineutrino energy), $\tau_{n}=886.7 \pm 1.9 \mathrm{~s}$ is the lifetime of the neutron, $f=1.686$ is the neutron statistical factor that includes Coulomb interactions of the final proton and electron, $m_{n}, m_{p}$ and $m_{e}$ are masses of the neutron, proton and electron, respectively.

In the Cowan and Reines experiment positrons and neutrons produced in the process (32) were detected and for the first time the corresponding very small neutrino cross section

$\sigma \sim 10^{-43} \mathrm{~cm}^{2}$ was measured. This became possible because of the existence of an intensive source of antineutrinos (reactor) and because of the invention of large scintillator counters.

The total cross section of the process (32) measured in the Cowan and Reines experiment,

$$
\sigma\left(\bar{\nu}_{e} p \rightarrow e^{+} n\right)_{\exp }=(11 \pm 4) \times 10^{-44} \mathrm{~cm}^{2},
$$


was in an agreement with the expected cross section

$$
\sigma\left(\bar{\nu}_{e} p \rightarrow e^{+} n\right)_{\mathrm{th}}=9.5 \times 10^{-44} \mathrm{~cm}^{2}
$$

The Cowan and Reines experiment was a crucial confirmation of the Pauli-Fermi hypothesis of existence of the neutrino. This experiment also confirmed the correctness of the fieldtheoretical relation (34) between the lifetime the neutron and the cross section of the crosssymmetrical process (32). However, since the energy of antineutrinos from a reactor is not enough to produce muons, the Cowan and Reines experiment could not reveal the existence of other flavor neutrinos, besides $\nu_{e}$.

The next flavor neutrino discovery experiment was the 1962 Brookhaven experiment of L.M. Lederman, M. Schwartz, J. Steinberger et al. [29].

At that time there were some indications that the muon neutrino (the neutrino that is produced in $\mu$-capture, $\mu$-decay and other weak processes in which the muon participates) and the electron neutrino are different particles. These indications were based on the comparison of the results of calculations of the probability of $\mu \rightarrow e+\gamma$ decay with the experimental upper bound for the probability of this decay.

If $\nu_{\mu}$ and $\nu_{e}$ are the same particle, the decay $\mu \rightarrow e+\gamma$ is allowed. The probability of this decay was calculated in Ref. [32] in the framework of a nonrenormalizable theory with intermediate $W$ boson (diagrams are similar to the diagrams in Fig. 1), assuming that the $W$-boson has a normal magnetic moment and that the cut-off mass is equal to the mass of the $W$. The resulting value of the ratio $R$ of the probability of the decay $\mu \rightarrow e+\gamma$ and the total probability of muon decay was $R=\alpha /(24 \pi) \simeq 10^{-4} 32$.

On the other hand, the decay $\mu \rightarrow e+\gamma$ was not observed experimentally. At the time of the Brookhaven experiment, the upper bound was $R \lesssim 10^{-8}$ [33, 34] ].

In spite of the indication of the existence of a muon neutrino given by the non-observation of $\mu \rightarrow e+\gamma$ decays, it was extremely important to check whether $\nu_{\mu}$ and $\nu_{e}$ are the same or different particles in a direct neutrino experiment. The Brookhaven experiment was the ideal experiment for this aims.

In this experiment the neutrino beam was produced in the decays of pions with a small admixture of neutrinos from the decays of kaons and muons. The dominant decay mode of the $\pi^{+}$meson is

$$
\pi^{+} \rightarrow \mu^{+}+\nu_{\mu}
$$

According to the universal $V-A$ theory of weak interactions of Feynman and Gell-Mann [36] and Marshak and Sudarshan [37, the ratio of the probability of the decay

$$
\pi^{+} \rightarrow e^{+}+\nu_{e}
$$

and the probability of the decay in Eq. (37) is aboutf $1.2 \times 10^{-4}$. Hence, the neutrino beam in the Brookhaven experiment was practically a pure beam of muon neutrinos.

\footnotetext{
${ }^{7}$ Now the upper bound is $R \leq 1.2 \times 10^{-11}$ 17.

8 The experiment was proposed by B. Pontecorvo in 1959 [35].

9 This prediction of the V-A theory was beautifully confirmed in a CERN experiment in 1958 [38].
} 
The pion beam in the experiment was produced by $15 \mathrm{GeV}$ protons striking a berillium target. Neutrinos from the decays of pions had a spectrum of energies $\lesssim 1 \mathrm{GeV}$. Neutrino interactions were observed in a 10 ton aluminum spark chamber.

According to field theory, a muon neutrinos produced in the decay (37) together with a $\mu^{+}$must produce $\mu^{-}$in the process

$$
\nu_{\mu}+N \rightarrow \mu^{-}+X .
$$

In order to investigate if $\nu_{\mu}$ and $\nu_{e}$ are the same or different particles, one needs to check whether $\nu_{\mu}$ 's can produce also electrons in the process

$$
\nu_{\mu}+N \rightarrow e^{-}+X .
$$

If electron and muon neutrinos are the same particles, according to the universal $V-A$ theory one must expect to observe in the detector approximately an equal number of electrons and muons.

In the Brookhaven experiment 34 single muon events have been observed, with an expected background from cosmic rays of 5 events. The measured cross section was in agreement with the $V-A$ theory. Six shower events were observed, with a distribution of sparks totally different from that expected for electrons. If $\nu_{\mu}$ and $\nu_{e}$ are the same particles, 29 electron events with energy more than $400 \mathrm{MeV}$ should have been observed in the experiment四.

Summarizing, the Brookhaven experiment proved that muon neutrinos, produced together with muons, cannot produce electrons in the process (40). Therefore, it was proved that $\nu_{e}$ and $\nu_{\mu}$ are different flavor neutrinos. The Brookhaven experiment also proved for the first time that accelerator $\nu_{\mu}$ 's produced in the process (37) can be detected.

Let us notice that the results of the Brookhaven experiment and all other data existing at that time were interpreted in terms of two conserved family lepton numbers $L_{e}$ and $L_{\mu}$ that allowed to distinguish $\left(\nu_{e}, e^{-}\right)$and $\left(\nu_{\mu}, \mu^{-}\right)$pairs and to forbid processes of type (40). We know now that in the framework of neutrino mixing family lepton numbers are not conserved and muon neutrinos at some distance can transform into electron neutrinos and produce electrons (as in the case of LSND experiment [8]). From the point of view of neutrino mixing, flavor neutrino discovery experiments require relatively small distances between neutrino sources and detector and relatively large energies, in order to satisfy the condition (29).

In 1975 the third lepton, $\tau$, was discovered by M. Perl et al. 441, 42]. After this discovery many decay modes of $\tau$ have been investigated:

$$
\begin{array}{ll}
\tau^{-} \rightarrow \mu^{-}+\bar{\nu}_{\mu}+\nu_{\tau}, & \tau^{-} \rightarrow \pi^{-}+\nu_{\tau}, \\
\tau^{-} \rightarrow e^{-}+\bar{\nu}_{e}+\nu_{\tau}, & \tau^{-} \rightarrow \pi^{-}+\pi^{0}+\nu_{\tau}, \\
& \tau^{-} \rightarrow K^{-}+\nu_{\tau},
\end{array}
$$

and others.

\footnotetext{
${ }^{10}$ In 1963 in CERN, with the invention of the magnetic horn, the intensity and purity of neutrino beams was greatly improved. The Brookhaven result was confirmed with good accuracy in a large 45 tons sparkchamber experiment [39] and in a large bubble chamber experiment 40].
} 
All experimental data on $\tau$ decays are in good agreement with the Standard Model 43, 44, 45. It is a general a consequence of field theory that the neutrino $\nu_{\tau}$ produced in $\tau$ decays as those in Eq. (41) can produce $\tau^{-}$'s in process as

$$
\nu_{\tau}+N \rightarrow \tau^{-}+X
$$

and others. Moreover, $e-\mu-\tau$ universality of weak interactions allows to predict the cross section of the process (42).

Therefore, the investigation of $\tau$ decays of the type (41) and subsequent charged-current processes as the one in Eq. (42) do not allow to check if $\nu_{\tau}$ is a new type of neutrino, different from $\nu_{\mu}$ and $\nu_{e}$. However, as in the case of $\nu_{\mu}$ and $\nu_{e}$ this can be tested in a different type of neutrino experiment.

In order to prove that $\nu_{\tau}$ is a new type of neutrino, it is necessary to prove either that $\nu_{\tau}$ 's cannot produce electrons or muons, or that $\nu_{\mu}$ 's and $\nu_{e}$ 's cannot produce $\tau$ 's.

The Brookhaven experiment proved that muon neutrinos produce muons and do not produce electrons with the predicted cross section. However, another type of experiment could prove that $\nu_{\mu}$ and $\nu_{e}$ are different particles. Imagine that it would be possible to create a pure beam of $\nu_{e}$ 's with energies well above of the threshold of $\mu^{-}$production. If in an experiment with such a beam it were shown that $\nu_{e}$ 's produce electrons and do not produce muons with the predicted cross section (under the assumption that $\nu_{\mu}$ and $\nu_{e}$ are the same particles) it would be proven that the flavor neutrinos $\nu_{\mu}$ and $\nu_{e}$ are different.

So far no experiment has proved that $\nu_{\tau}$ 's cannot produce electrons or muons, but several neutrino oscillation experiments looking for $\nu_{\mu} \rightarrow \nu_{\tau}$ and $\nu_{e} \rightarrow \nu_{\tau}$ transitions have proved that $\nu_{\mu}$ 's and $\nu_{e}$ 's cannot produce $\tau$ 's. These experiments are: FNAL-E531 $\left(\nu_{\mu} \rightarrow \nu_{\tau}\right.$ and $\left.\nu_{e} \rightarrow \nu_{\tau}\right)$ 46], CHARM II $\left(\nu_{\mu} \rightarrow \nu_{\tau}\right)$ 47], CCFR $\left(\nu_{e} \rightarrow \nu_{\tau}\right)$ [4], CHORUS $\left(\nu_{\mu} \rightarrow \nu_{\tau}\right.$ and $\left.\nu_{e} \rightarrow \nu_{\tau}\right)$ 49] NOMAD $\left(\nu_{\mu} \rightarrow \nu_{\tau}\right.$ and $\left.\nu_{e} \rightarrow \nu_{\tau}\right)$ [50.

For example, the neutrino beam in the recent CHORUS and NOMAD experiments, produced with the CERN SPS accelerator, was predominantly composed of $\nu_{\mu}$ 's, with small $\bar{\nu}_{\mu}, \nu_{e}$ and $\bar{\nu}_{e}$ components. The percentage of $\nu_{e}$ was about $0.9 \%$ and the contamination of $\nu_{\tau}$ in the beam is negligible $\left(\simeq 5 \times 10^{-6}\right)$. The average energies of $\nu_{\mu}$ and $\nu_{e}$ are $27 \mathrm{GeV}$ and $40 \mathrm{GeV}$, respectively. Notice that the threshold of production of $\tau$ 's in the process (42) is $3.5 \mathrm{GeV}$.

No event of $\tau$-lepton production have been observed in the CHORUS and NOMAD experiments at a distance of about $600 \mathrm{~m}$ from the source, leading to the following upper bounds for the probabilities of $\nu_{\mu} \rightarrow \nu_{\tau}$ and $\nu_{e} \rightarrow \nu_{\tau}$ transitions:

$$
\begin{array}{lll}
P_{\nu_{\mu} \rightarrow \nu_{\tau}} \leq 3.4 \times 10^{-4}, & & P_{\nu_{e} \rightarrow \nu_{\tau}} \leq 2.6 \times 10^{-2} \\
P_{\nu_{\mu} \rightarrow \nu_{\tau}} \leq 2.1 \times 10^{-3}, & & P_{\nu_{e} \rightarrow \nu_{\tau}} \leq 2.6 \times 10^{-2}
\end{array}
$$

These very stringent limits imply that the flavor neutrinos $\nu_{\mu}$ and $\nu_{e}$ are different from $\nu_{\tau}$. If $\nu_{\mu}$ and $\nu_{\tau}$ were the same particle, about 5014 one- $\mu$ events (events with one reconstructed $\mu^{-}$ from the decay $\tau^{-} \rightarrow \mu^{-}+\bar{\nu}_{\mu}+\nu_{\tau}$ ) would have been observed in the CHORUS experiment. In reality no event of this type was observed. If $\nu_{e}$ and $\nu_{\tau}$ were the same particle, about 23 events with a highly energetic $e^{-}$from the decay $\tau^{-} \rightarrow e^{-}+\bar{\nu}_{e}+\nu_{\tau}$ would have been observed in the NOMAD experiment. No event of this type was observed above the expected background. 
Let us notice that also the experimental upper bounds for the relative probabilities of the decays $\tau^{-} \rightarrow \mu^{-}+\gamma$ and $\tau^{-} \rightarrow e^{-}+\gamma$,

$$
R_{\mu} \leq 1.1 \times 10^{-6}, \quad R_{e} \leq 2.7 \times 10^{-6}[17
$$

imply that $\nu_{\tau}$ is different from $\nu_{e}$ and $\nu_{\mu}$. This follows from an argument that is similar to the one explained above for the decay $\mu \rightarrow e+\gamma$ at the time of the Brookhaven experiment, based on the smallness of the upper limits (44) with respect to the value $R \simeq 10^{-4}$ expected if $\nu_{\mu}$ and $\nu_{\tau}$ or $\nu_{e}$ and $\nu_{\tau}$ are the same particle [32].

The flavor neutrino $\nu_{\tau}$ was directly detected for the first time in the DONUT experiment [30]. The DONUT experiment is a beam-dump experiment. Neutrinos in this experiment were produced in the decays of short-lived charm particles. The neutrino beam was composed mainly of $\nu_{e}$ 's and $\nu_{\mu}$ 's, with about $5 \%$ of $\nu_{\tau}$ 's from the decay

$$
D_{s} \rightarrow \tau+\nu_{\tau}
$$

Neutrinos are detected in the DONUT experiment in emulsions at a distance of $36 \mathrm{~m}$ from the source. The important signature of $\tau$ production is the kink from $\tau$-decay. In a set of 203 neutrino interactions, four events with a kink, which satisfy all requirements for the production and decay of $\tau$, were found. The estimated background is $0.34 \pm 0.05$ events.

Up to now we considered only CC processes due to the intermediate $W$-boson. The investigation of $\mathrm{NC}$ processes due to the intermediate $Z$-boson, as

$$
\nu_{\mu}+N \rightarrow \nu_{\mu}+X, \quad \nu_{\mu}+e \rightarrow \nu_{\mu}+e, \quad \bar{\nu}_{e}+e \rightarrow \bar{\nu}_{e}+e
$$

and others, have allowed to prove that $\nu_{\mu}$ and $\nu_{e}$ interact with the $Z$-boson in accordance with the Standard Model.

The four LEP experiments (ALEPH, DELPHI, L3, OPAL) determined with high accuracy that the number $n_{\nu}$ of light flavor neutrinos (mass $\lesssim 45 \mathrm{GeV}$ ) produced in the decay of the $Z$-boson is three (see [17]):

$$
n_{\nu}=3.00 \pm 0.06
$$

From the observation of the processes (46) it follows that two flavor neutrinos that contribute to $n_{\nu}$ in Eq. (47) are $\nu_{\mu}$ and $\nu_{e}$. The most plausible candidate for the third neutrino is $\nu_{\tau}$, discovered in $\mathrm{CC}$ reactions. It is interesting that we still have no direct proof of that (for such a proof the investigation of $\nu_{\tau}$-induced $\mathrm{NC}$ processes is required).

\section{Lepton number and neutrino mixing}

In the case of neutrino mixing, the possible existence of a conserved lepton number can be connected only with neutrinos with definite masses. The neutrino mass term has the form

$$
\mathcal{L}_{\text {mass }}=-\sum_{i} m_{i} \overline{\nu_{i R}} \nu_{i L}+\text { h.c. . }
$$


If the right-handed components $\nu_{i R}$ and the left-handed components $\nu_{i L}$ are independent, the massive neutrinos $\nu_{i}$ are Dirac particles. Indeed, in this case the total Lagrangian is invariant under the global gauge transformation

$$
\nu_{i L} \rightarrow e^{i \alpha} \nu_{i L}, \quad \nu_{i R} \rightarrow e^{i \alpha} \nu_{i R}, \quad \ell \rightarrow e^{i \alpha} \ell,
$$

where $\alpha$ is an arbitrary constant. This invariance implies that the lepton number $L$, which has the same value for $e^{-}, \mu^{-}, \tau^{-}$and all $\nu_{i}$ 's, is conserved. In this case the quanta of the fields $\nu_{i}$ are neutrinos with $L=1$ and antineutrinos with $L=-1$.

On the other hand, if the right-handed components $\nu_{i R}$ and the left-handed components $\nu_{i L}$ are not independent, but connected by the relation

$$
\nu_{i R}=\left(\nu_{i L}\right)^{c}=\mathcal{C}\left(\overline{\nu_{i L}}\right)^{T}
$$

( $\mathcal{C}$ is the matrix of charge conjugation), the massive neutrinos $\nu_{i}$ are Majorana particles. In this case there is no any gauge invariance of the total Lagrangian $\square$ and the neutrino field $\nu_{i}=\nu_{i L}+\nu_{i R}$ satisfies the Majorana condition

$$
\nu_{i}=\left(\nu_{i}\right)^{c} .
$$

This condition implies that the quanta of the field $\nu_{i}$ are truly neutral Majorana neutrinos (identical to antineutrinos).

The problem of the nature of neutrino with definite masses is one of the most fundamental problem of the physics of massive and mixed neutrinos and is connected with the origin of neutrino masses and neutrino mixing.

Dirac neutrino masses can be generated by the standard Higgs mechanism. Majorana neutrino masses require a new mechanism of neutrino mass generation that is beyond the Standard Model. One of the most popular mechanisms of neutrino mass generation is the see-saw mechanism [51, 52, 53]. This mechanism is based on the assumption that the law of conservation of lepton number is violated at a scale that is much larger then the scale of violation of the electroweak symmetry. The see-saw mechanism allows to connect the smallness of neutrino masses with a large physical scale that characterizes the violation of the lepton number conservation law.

In order to reveal the Dirac or Majorana nature of neutrinos it is necessary to study neutrino mass effects: T2. $^{2}$.

It is impossible to distinguish massive Dirac and Majorana neutrinos through the investigation of neutrino oscillations [55, 56, 57. Indeed, in the case of neutrino mixing the leptonic CC current has the form

$$
j_{\alpha}^{\mathrm{CC}}=2 \sum_{\ell, i} \overline{\ell_{L}} \gamma_{\alpha} U_{\ell i} \nu_{i L} .
$$

\footnotetext{
${ }^{11}$ In the Majorana case the transformation $\nu_{i L} \rightarrow e^{i \alpha} \nu_{i L}$ requires $\nu_{i R} \rightarrow e^{-i \alpha} \nu_{i R}$. It is obvious that the mass term (48) is not invariant under these transformations.

${ }^{12}$ Dirac neutrinos and Majorana neutrinos are different only if neutrino masses are different from zero. In the case of standard electroweak interactions with left-handed neutrino fields there is no physical difference between massless Dirac and massless Majorana neutrinos [54].
} 
If $\nu_{i}$ are Dirac fields, the mixing matrix $U$ is determined up to the transformation

$$
U_{\ell i} \rightarrow e^{-i \alpha_{\ell}} U_{\ell i} e^{i \beta_{i}},
$$

where $\alpha_{\ell}$ and $\beta_{i}$ are arbitrary parameters. This is due to the fact that the phases of Dirac fields are arbitrary.

In the case of Majorana neutrinos, the Majorana condition (51) does not allow to include arbitrary phases into the fields. Thus, in the Majorana case the mixing matrix $U$ is determined only up to the transformation

$$
U_{\ell i} \rightarrow e^{-i \alpha_{\ell}} U_{\ell i},
$$

From Eqs. (53) and (54) it follows that the number of physical CP-violating phases in the Dirac and Majorana cases are different

From Eq. (17) one can see that under both transformation (53) and (54) the amplitude of $\nu_{\ell} \rightarrow \nu_{\ell^{\prime}}$ transitions is transformed as

$$
\mathcal{A}_{\nu_{\ell} \rightarrow \nu_{\ell^{\prime}}} \rightarrow e^{-i\left(\alpha_{\ell^{\prime}}-\alpha_{\ell}\right)} \mathcal{A}_{\nu_{\ell} \rightarrow \nu_{\ell^{\prime}}},
$$

and the probability of $\nu_{\ell} \rightarrow \nu_{\ell^{\prime}}$ transitions is invariant under the transformation (55). This means that the transition probability is independent from the additional phases in the Majorana case.

The most promising process that allows to investigate the nature of massive neutrino (Dirac or Majorana?) is neutrinoless double- $\beta$ decay of even-even nuclei:

$$
\mathcal{N}(A, Z) \rightarrow \mathcal{N}(A, Z+2)+e^{-}+e^{-} .
$$

The diagram of this process is depicted in Fig. 2. In the case of mixing of Majorana neutrinos, the neutrino propagator in Fig. 2 is given by

$$
\begin{aligned}
\left\langle 0\left|T\left[\nu_{e L}\left(x_{1}\right) \nu_{e L}^{T}\left(x_{2}\right)\right]\right| 0\right\rangle & =-\sum_{i} U_{e i}^{2} \frac{1-\gamma_{5}}{2}\left\langle 0\left|T\left[\nu_{i}\left(x_{1}\right) \bar{\nu}_{i}\left(x_{2}\right)\right]\right| 0\right\rangle \frac{1-\gamma_{5}}{2} \mathrm{C} \\
& =-\frac{i}{(2 \pi)^{4}} \int \mathrm{d}^{4} p e^{-i p\left(x_{1}-x_{2}\right)} \sum_{i} U_{e i}^{2} \frac{m_{i}}{p^{2}-m_{i}^{2}} \frac{1-\gamma_{5}}{2} \mathcal{C} .
\end{aligned}
$$

From Eq. (57) it follows that in the case of small neutrino masses the matrix element of neutrinoless double- $\beta$ decay is proportional to

$$
\langle m\rangle=\sum_{i} U_{e i}^{2} m_{i} .
$$

\footnotetext{
${ }^{13}$ For $n$ families the number of physical phases in the case of Dirac neutrinos is $(n-1)(n-2) / 2$ (in this case the number of phases is the same as in the quark case). In the case of Majorana neutrinos the number of the physical phases is larger: $n(n-1) / 2$, i.e. there are $n-1$ additional phases.
} 
Thus, the process (56) is allowed if neutrinos are Majorana particles and massive. Notice that neutrino mixing is not required for that

Neutrinoless double- $\beta$ is a process of second order in the Fermi constant $G_{F}$. Its matrix element is proportional to small neutrino masses. The expected lifetime of neutrinoless double- $\beta$ decay is much larger than the lifetime of usual $\beta$-decays. However, because of the clear signature of the process (two electrons with definite total energy in the final state), several experiments have obtained very large lower bounds for the lifetime of neutrinoless double- $\beta$ decay of different nuclei (see [58]). The lower limits for the lifetimes of ${ }^{76} \mathrm{Ge}$ and ${ }^{136}$ Xe obtained in the Heidelberg-Moscow experiment [59] and in the Gotthard experiment [60] are

$$
T\left({ }^{76} \mathrm{Ge}\right) \geq 5.7 \times 10^{25} \mathrm{yr}, \quad T\left({ }^{136} \mathrm{Xe}\right) \geq 4.4 \times 10^{23} \mathrm{yr}, \quad \text { at } 90 \% \mathrm{CL} .
$$

These limits imply upper bounds for the effective Majorana mass $\langle m\rangle$, with values that depend on the calculation of nuclear matrix elements. The results of different calculations lead to the limits

$$
\langle m\rangle \leq 0.2-0.6 \mathrm{eV}\left({ }^{76} \mathrm{Ge}\right), \quad\langle m\rangle \leq 2.2-5.2 \mathrm{eV}\left({ }^{136} \mathrm{Xe}\right), \quad \text { at } 90 \% \mathrm{CL} .
$$

Several new experiments searching for neutrinoless double- $\beta$ decay are in preparation. These future experiments are planned to be sensitive to values of $\langle m\rangle \sim 10^{-1} \mathrm{eV}$ [61, 62], or even $\sim 10^{-2} \mathrm{eV}$ 63, 64, 65].

The results of neutrino oscillation experiments give information on neutrino masses and on the elements of the neutrino mixing matrix. The possible values of $|\langle m\rangle|$, depend, among others, on the character of the neutrino mass spectrum, on the real existence of the oscillations observed in the LSND experiment, and on the absolute values of neutrino masses (see [66, 67, 68, 69, 70, 71]).

If the results of the LSND experiment will not be confirmed by future experiments, all the other neutrino data can be explained by the existence of only three massive and mixed neutrinos.

If there is a hierarchy of neutrino masses,

$$
m_{1} \ll m_{2} \ll m_{3},
$$

there is a stringent upper bound for the effective Majorana mass [67, 69]:

$$
|\langle m\rangle| \lesssim 10^{-2} \mathrm{eV} .
$$

14 The probability of neutrinoless double- $\beta$ decay is suppressed because of the smallness of neutrino masses. If there is no mixing (i.e. $U$ is the unit matrix), $\langle m\rangle=m$, where $m$ is the Majorana mass of $\nu_{e}$. In neutron decay both right-handed and left-handed Majorana electron neutrinos are emitted together with $e^{- \text {'s. The }}$ amplitude of the production of left-handed neutrinos is proportional to $\mathrm{m} / E$ ( $E$ is the neutrino energy). The left-handed Majorana neutrino can be absorbed by another neutron in a nucleus with the production of another $e^{-}$. The amplitude of absorption of right-handed neutrinos is proportional to $m / E$. Hence, the amplitude of neutrinoless double $\beta$-decay is proportional to $(m / E) \lesssim 10^{-7}$ for typical nuclear energies $E \sim 10 \mathrm{MeV}$. 
In the case of an "inverted hierarchy",

$$
m_{1} \ll m_{2}<m_{3}, \quad m_{1} \ll 1 \mathrm{eV}
$$

the upper bound for the effective Majorana mass is less stringent 69]:

$$
|\langle m\rangle| \lesssim 7 \times 10^{-2} \mathrm{eV}
$$

If the evidence in favor of short-baseline $\nu_{\mu} \rightarrow \nu_{e}$ oscillations obtained in the LSND experiment will be confirmed by other experiments, the effective Majorana mass could be as large as $\sim 1 \mathrm{eV}$ or smaller than $\sim 10^{-2} \mathrm{eV}$, depending on the neutrino mass spectrum [66, 67, 68, 69, 70].

\section{Conclusions}

We have discussed the notion of lepton numbers in the case of neutrino mixing. We have stressed that the existence of neutrino oscillations means that there are no conserved family lepton numbers $L_{e}, L_{\mu}, L_{\tau}$. The flavor neutrinos $\nu_{e}, \nu_{\mu}$ and $\nu_{\tau}$ participate in weak interactions ( $\nu_{\mu}$ is produced together with a $\mu^{+}$in $\pi^{+}$decay, etc.). In the case of small neutrino masses the states of flavor neutrinos are superpositions of the states of neutrinos $\nu_{i}$ with definite masses. Flavor neutrinos are not quanta of any field and they have no definite masses.

We have discussed the difference between different flavor neutrino discovery experiments. The results of $\nu_{\mu} \rightarrow \nu_{\tau}$ and $\nu_{e} \rightarrow \nu_{\tau}$ oscillation experiments clearly demonstrate that $\nu_{\tau}$ is a new type of flavor neutrino, different from $\nu_{e}$ and $\nu_{\mu}$. The $\nu_{\tau}$ has been detected directly in the recent DONUT experiment.

We have stressed that a conserved lepton number $L$ can exist only if massive neutrinos are Dirac particles. In this case the electron, muon, tau-lepton and massive neutrinos $\nu_{i}$ have the same values of $L$. The lepton number $L$ distinguishes neutrinos from antineutrinos. Different neutrinos differ by the value of their masses.

If massive neutrinos are Majorana particles there are no conserved lepton numbers. The search for neutrinoless double- $\beta$ decay is the most promising method to test the conservation of lepton number in the case of neutrino mixing.

\section{Acknowledgments}

We would like to thank W. Grimus for useful comments on a preliminary version of this paper. We would also like to thank W. Alberico and A. Bottino for useful discussions.

\section{References}

[1] S. Fukuda et al. (Super-Kamiokande Coll.), hep-ex/0009001; T. Kajita (SuperKamiokande Coll.), Talk presented at NOW2000, Otranto, Italy, September 2000 (http://www.ba.infn.it/now2000).

[2] B. T. Cleveland et al., Astrophys. J. 496, 505 (1998). 
[3] Kamiokande, Y. Fukuda et al., Phys. Rev. Lett. 77, 1683 (1996).

[4] GALleX, W. Hampel et al., Phys. Lett. B447, 127 (1999).

[5] SAGE, J. N. Abdurashitov et al., Phys. Rev. C60, 055801 (1999), astro-ph/9907113.

[6] Y. Suzuki (Super-Kamiokande Coll.), Talk presented at Neutrino 2000, Sudbury, Canada, 16-21 June 2000 (http://nu2000.sno.laurentian.ca); Talk presented at NOW2000, Otranto, Italy, September 2000 (http://www.ba.infn.it/^now2000).

[7] GNO, M. Altmann et al., (2000), hep-ex/0006034.

[8] G. Mills (LSND Coll.), Talk presented at Neutrino 2000, Sudbury, Canada, 16-21 June 2000 (http://nu2000.sno.laurentian.ca).

[9] S. M. Bilenky, C. Giunti, and W. Grimus, Prog. Part. Nucl. Phys. 43, 1 (1999), hep$\mathrm{ph} / 9812360$.

[10] S. T. Petcov, Sov. J. Nucl. Phys. 25, 340 (1977), Erratum, ibid. 25, 698 (1977).

[11] T. P. Cheng and L.-F. Li, Phys. Rev. Lett. 45, 1908 (1980).

[12] S. M. Bilenky and S. T. Petcov, Rev. Mod. Phys. 59, 671 (1987).

[13] R. Barbieri and L. J. Hall, Phys. Lett. B338, 212 (1994), hep-ph/9408406.

[14] R. Barbieri, L. Hall, and A. Strumia, Nucl. Phys. B445, 219 (1995), hep-ph/9501334.

[15] N. Arkani-Hamed, H.-C. Cheng, and L. J. Hall, Phys. Rev. D53, 413 (1996), hep$\mathrm{ph} / 9508288$.

[16] J. Hisano, T. Moroi, K. Tobe, and M. Yamaguchi, Phys. Lett. B391, 341 (1997), hep-ph/9605296.

[17] D. E. Groom et al., Eur. Phys. J. C15, 1 (2000), WWW page: http://pdg.lbl.gov.

[18] S. M. Bilenky and B. Pontecorvo, Phys. Rept. 41, 225 (1978).

[19] C. W. Kim and A. Pevsner, Neutrinos in physics and astrophysics (Harwood Academic Press, Chur, Switzerland, 1993), Contemporary Concepts in Physics, Vol. 8.

[20] C. Giunti and C. W. Kim, (2000), hep-ph/0011074.

[21] L.B. Okun, Surveys High Energy Physics 15, 75 (2000).

[22] W. Grimus, S. Mohanty, and P. Stockinger, (1999), hep-ph/9909341.

[23] C. Giunti, C. W. Kim, and U. W. Lee, Phys. Rev. D44, 3635 (1991).

[24] C. Giunti and C. W. Kim, Phys. Rev. D58, 017301 (1998), hep-ph/9711363. 
[25] C. Giunti, C. W. Kim, and U. W. Lee, Phys. Rev. D45, 2414 (1992).

[26] F. Reines and C. L. Cowan, Phys. Rev. 92, 830 (1953).

[27] C. L. Cowan, F. Reines, F. B. Harrison, H. W. Kruse, and A. D. McGuire, Science 124, 103 (1956).

[28] F. Reines and C. L. Cowan, Phys. Rev. 113, 273 (1959).

[29] G. Danby et al., Phys. Rev. Lett. 9, 36 (1962).

[30] DONUT, K. Kodama et al., (2000), hep-ex/0012035.

[31] E.J. Konopinski, The Theory of Beta Radioactivity (Clarendon, Oxford, 1966).

[32] G. Feinberg, Phys. Rev. 110, 1482 (1958).

[33] D. Bartlett et al., Phys. Rev. Lett. 8, 120 (1962).

[34] S. Frankel et al., Phys. Rev. Lett. 8, 123 (1962).

[35] B. Pontecorvo, Sov. Phys. JETP 37, 1236 (1960).

[36] R. P. Feynman and M. Gell-Mann, Phys. Rev. 109, 193 (1958).

[37] E. C. G. Sudarshan and R. e. Marshak, Phys. Rev. 109, 1860 (1958).

[38] T. Fazzini, G. Fidecaro, A. W. Merrison, H. Paul, and A. V. Tollestrup, Phys. Rev. Lett. 1, 247 (1958).

[39] J. K. Bienlein et al., Phys. Lett. 13, 80 (1964).

[40] M. M. Block et al., Phys. Lett. 12, 281 (1964).

[41] M. L. Perl et al., Phys. Rev. Lett. 35, 1489 (1975).

[42] M. L. Perl, Ann. Rev. Nucl. Part. Sci. 30, 299 (1980).

[43] A. Pich, (2000), hep-ph/0012297.

[44] M. L. Perl, (1998), hep-ph/9812400.

[45] A. Stahl, Physics with tau leptons (Springer, Berlin, Germany, 2000).

[46] FERMilab E531, N. Ushida et al., Phys. Rev. Lett. 57, 2897 (1986).

[47] CHARM-II, M. Gruwe et al., Phys. Lett. B309, 463 (1993).

[48] CCFR/NuTeV, D. Naples et al., Phys. Rev. D59, 031101 (1999), hep-ex/9809023.

[49] CHORUS, E. Eskut et al., Phys. Lett. B497, 8 (2001). 
[50] NOMAD, P. Astier et al., Phys. Lett. B483, 387 (2000).

[51] M. Gell-Mann, P. Ramond and R. Slansky, in Supergravity, p. 315, edited by F. van Nieuwenhuizen and D. Freedman, North Holland, Amsterdam, 1979.

[52] T. Yanagida, Proc. of the Workshop on Unified Theory and the Baryon Number of the Universe, KEK, Japan, 1979.

[53] R. N. Mohapatra and G. Senjanovic, Phys. Rev. Lett. 44, 912 (1980).

[54] R. E. Marshak, Riazuddin, and C. P. Ryan, Theory of weak interactions in particle physics (Wiley-Interscience, 1969).

[55] S. M. Bilenky, J. Hosek, and S. T. Petcov, Phys. Lett. B94, 495 (1980).

[56] M. Doi, T. Kotani, H. Nishiura, K. Okuda, and E. Takasugi, Phys. Lett. B102, 323 (1981).

[57] P. Langacker, S. T. Petcov, G. Steigman, and S. Toshev, Nucl. Phys. B282, 589 (1987).

[58] V. I. Tretyak and Y. G. Zdesenko, Atomic Data and Nuclear Data Tables 61, 43 (1995).

[59] L. Baudis et al., Phys. Rev. Lett. 83, 41 (1999), hep-ex/9902014.

[60] R. Luescher et al., Phys. Lett. B434, 407 (1998).

[61] A. Alessandrello et al., Nucl. Phys. Proc. Suppl. 87, 78 (2000).

[62] NEMO, X. Sarazin and D. Lalanne, (2000), hep-ex/0006031.

[63] H. V. Klapdor-Kleingrothaus, J. Hellmig, and M. Hirsch, J. Phys. G24, 483 (1998).

[64] G. Bellini et al., Phys. Lett. B493, 216 (2000).

[65] M. Danilov et al., Phys. Lett. B480, 12 (2000), hep-ex/0002003.

[66] S. M. Bilenky, C. Giunti, C. W. Kim, and S. T. Petcov, Phys. Rev. D54, 4432 (1996), hep-ph/9604364.

[67] S. M. Bilenky, C. Giunti, C. W. Kim, and M. Monteno, Phys. Rev. D57, 6981 (1998), hep-ph/9711400.

[68] C. Giunti, Phys. Rev. D61, 036002 (2000), hep-ph/9906275.

[69] S. M. Bilenky, C. Giunti, W. Grimus, B. Kayser, and S. T. Petcov, Phys. Lett. B465, 193 (1999), hep-ph/9907234.

[70] H. V. Klapdor-Kleingrothaus, H. Pas, and A. Y. Smirnov, (2000), hep-ph/0003219.

[71] S. M. Bilenky, S. Pascoli, and S. T. Petcov, (2001), hep-ph/0102265. 

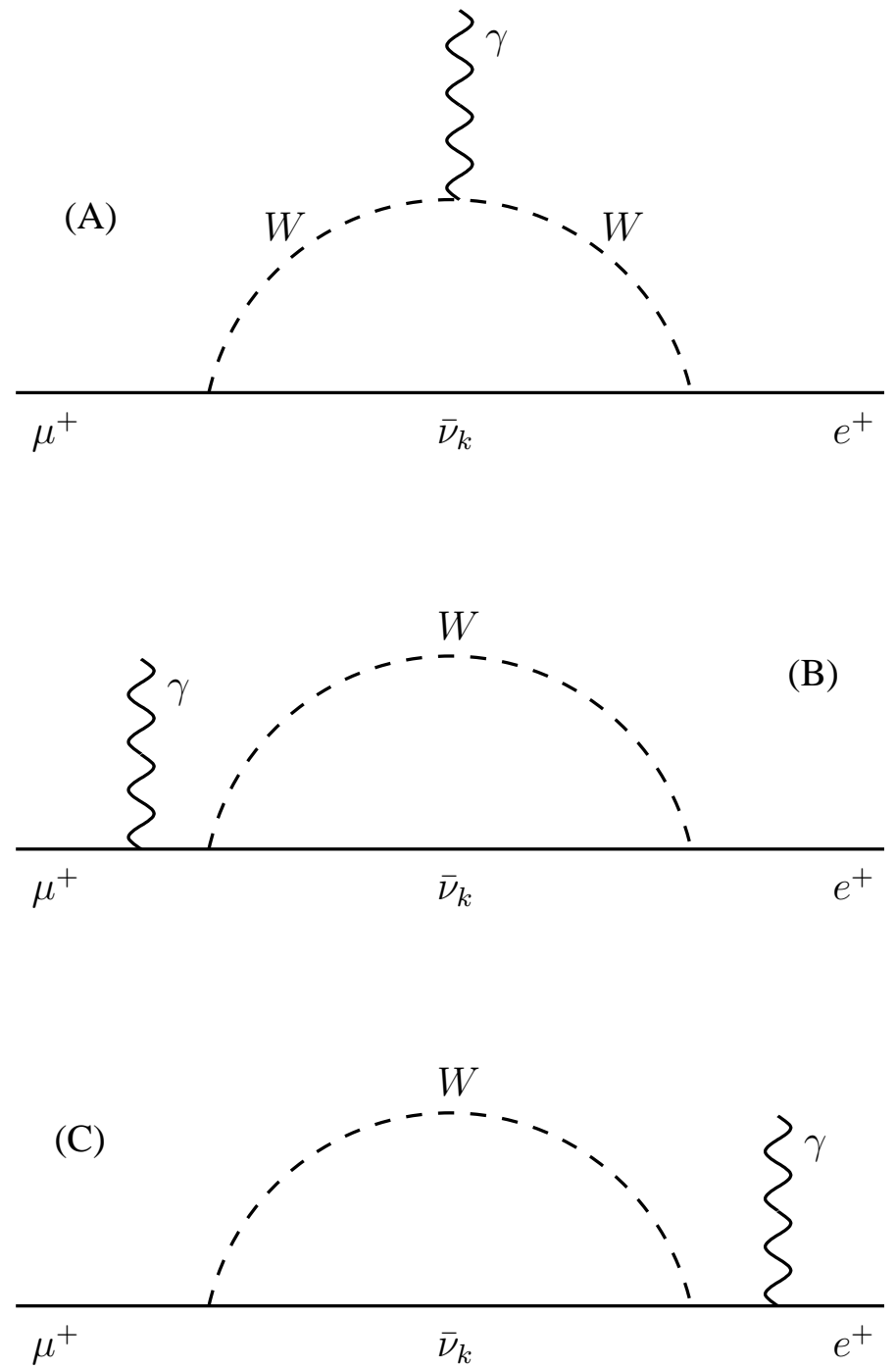

Figure 1: Diagrams contributing to the decay $\mu^{+} \rightarrow e^{+}+\gamma$ at lowest order. 


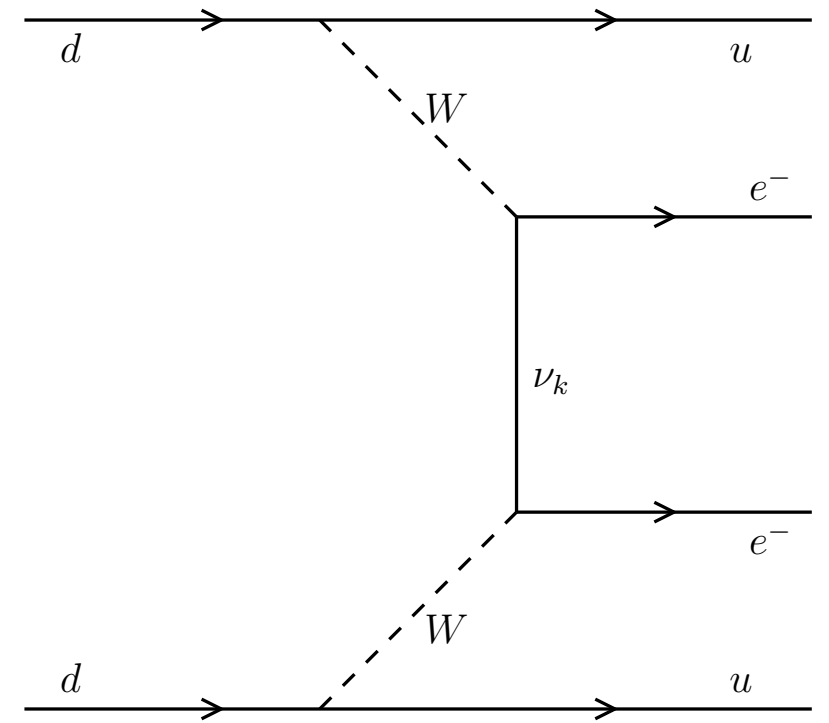

Figure 2: Diagram of neutrinoless double- $\beta$ decay. 